\title{
Comparison of the Hyponatremic Effects of Erythropoietin and U-74389G
}

\section{Constantinos Tsompos ${ }^{1 *}$, Constantinos Panoulis², Konstantinos Toutouzas ${ }^{3}$, Aggeliki Triantafyllou, George C Zografos ${ }^{3}$, Kalliopi Tsarea ${ }^{5}$, Maria Karamperi ${ }^{5}$ and Apostolos Papalois ${ }^{6}$}

${ }^{1}$ Department of Gynecology, General Hospital of Thessaloniki "St. Dimitrios" Thessaloniki, Greece

${ }^{2}$ Department of Obstetrics \& Gynecology, Aretaieion Hospital, Athens University, Greece

${ }^{3}$ Department of Surgery, Ippokrateion General Hospital, Athens University, Greece

${ }^{4}$ Department of Biologic Chemistry, Athens University, Greece

${ }^{5}$ Experimental Research Centre ELPEN Pharmaceuticals, S.A. Inc., Co., Greece

${ }^{6}$ Experimental, Educational and Research Center ELPEN, School of Medicine, European University Cyprus, Greece

\begin{abstract}
Aim: This study calculated the effects on serum sodium ( $\mathrm{Na}$ ) levels, after treatment with either of 2 drugs: The erythropoietin (Epo) and the antioxidant lazaroid (L) drug U-74389G. The calculation was based on the results of 2 preliminary studies, each one of which estimated the certain influence, after the respective drug usage in an induced ischemia reperfusion (IR) animal experiment.

Materials and methods: The 2 main experimental endpoints at which the serum Na levels were evaluated was the $60^{\text {th }}$ reperfusion $\min$ (for the groups $A, C$ and $E$ ) and the $120^{\text {th }}$ reperfusion min (for the groups $B, D$ and $F$ ). Specially, the groups $A$ and $B$ were processed without drugs, groups $C$ and $D$ after Epo administration; whereas groups $E$ and $F$ after the $L$ administration.

Results: The first preliminary study of Epo presented a non significant hyponatremic effect by $0.11 \% \pm 0.38 \%$ ( $p$-value = 0.7531 ). The second preliminary study of U-74389G presented a non significant hyponatremic effect by $0.32 \% \pm 0.36 \%$ $(p$-value $=0.3693)$. These 2 studies were co-evaluated since they came from the same experimental setting. The outcome of the co-evaluation was that $L$ is 2.74914 -fold [2.74424-2.754048] more hyponatremic than Epo ( $p$-value $=0.0000)$.

Conclusions: The anti-oxidant capacities of U-74389G ascribe 2.74914-fold more hyponatremic effects than Epo ( $p$-value $=0.0000$ ).
\end{abstract}

Keywords

Ischemia, Erythropoietin, U-74389G, Serum sodium levels, Reperfusion

\section{Introduction}

The lazaroid U-74389G (L) may be not famous for its hyponatremic [1] capacity ( $p$-value $=0.3693$ ). U-74389G as a novel antioxidant factor, implicates exactly only 260 published studies. The ischemia reperfusion (IR) type of experiments was noted in $18.84 \%$ of these studies. A tissue protective feature of U-74389G was obvious in these IR studies. The U-74389G chemically known as 21-[4-(2,6-di-1-pyrrolidinyl-4pyrimidinyl)-1-piperazinyl]-pregna-1,4,9(11)-triene-3,20-dione male ate salt is an antioxidant complex, which prevents the lipid peroxidation either iron-dependent, or arachidonic acid-induced one. Animal kidney, liver, brain microvascular endothelial cells monolayers and heart models were protected by U-74389G after IR injury. U-74389G also attenuates the leukocytes; down-regulates the proinflammatory gene; treats the endotoxin shock; produces cytokine; enhances the mononuclear immunity; protects the endothelium and presents antishock property.

Erythropoietin (Epo) even if is not famous for its hypona-

*Corresponding author: Constantinos Tsompos, Department of Gynecology, General Hospital of Thessaloniki "St. Dimitrios" 2 Elenis Zografou Street, Thessaloniki 54634, Hellas, Greece, Tel: 00302313322171; 00306946674264, Fax: 00302106811215

Accepted: August 08, 2019

Published online: August 10, 2019

Citation: Tsompos C, Panoulis C, Toutouzas K, et al. (2019) Comparison of the Hyponatremic Effects of Erythropoietin and U-74389G. Insights Biomed Res 3(1):52-55 
tremic [2] action ( $p$-value $=0.7531$ ), it can be used as a reference drug for comparison with U-74389G. Although Epo is met in over 30,844 published biomedical studies, only a $3.62 \%$ of them negotiate the known type of IR experiments. Nevertheless, Epo as a cytokine, it is worth of being studied about its effects on serum sodium ( $\mathrm{Na}$ ) levels too. This experimental work tried to compare the effects of the above drugs on a rat induced IR protocol. They were tested by calculating the serum $\mathrm{Na}$ levels alterations.

\section{Materials and Methods}

\section{Animal preparation}

The Vet licenses under 3693/12-11-2010 \& 14/10-1-2012 numbers, the granting company and the experiment location are mentioned in preliminary references $[1,2]$. The human animal care of Albino female Wistar rats, the 7 days pre-experimental ad libitum diet, the non-stop intra-experimental anesthesiologic techniques, the acidometry, the electrocardiogram, the oxygen supply and post-experimental euthanasia are also described in preliminary references. Rats were 16-18 weeks old. They were randomly assigned to six (6) groups consisted in $\mathrm{N}=10$. The stage of 45 min hypoxia was common for all 6 groups. Afterwards, reperfusion of $60 \mathrm{~min}$ was followed in group $A$; reperfusion of 120 min in group $B$; immediate Epo intravenous (IV) administration and reperfusion of $60 \mathrm{~min}$ in group C; immediate Epo IV administration and reperfusion of $120 \mathrm{~min}$ in group D; immediate U-74389G IV administration and reperfusion of $60 \mathrm{~min}$ in group $\mathrm{E}$; and immediate U-74389G IV administration and reperfusion of 120 min in group $F$ (Table 1). The dose height assessment for both drugs are described at preliminary studies as $10 \mathrm{mg} / \mathrm{Kg}$ body mass.

Ischemia was caused by laparotomic clamping the inferior aorta over renal arteries with forceps for $45 \mathrm{~min}$. The clamp removal was restoring the inferior aorta patency and reperfusion. After the blood flow interruption, the protocol of IR was applied, as described above for each experimental group. The drugs were administered at the time of reperfusion; through inferior vena cava catheter. The Na levels were determined at $60^{\text {th }}$ min of reperfusion (for $A, C$ and $E$ groups) and at $120^{\text {th }}$ min of reperfusion (for $B, D$ and $F$ groups). Along, a weak relation was rised between Na values with animals' mass ( $p$-value $=0.2366$ ).

Table 1: Serum sodium levels at the rest endpoints of $1 \mathrm{~h}, 1.5 \mathrm{~h}$, and $2.0 \mathrm{~h}$.

\begin{tabular}{|l|l|l|l|l|l|}
\hline Sodium levels & Obs & Mean & Std. Dev. & Min & Max \\
\hline 1 h placebo & 10 & 137.8 & 2.299758 & 135 & 141 \\
\hline 1.5 h placebo & 10 & 137.6 & 2.157674 & 134.5 & 140.5 \\
\hline 2 h placebo & 10 & 137.4 & 2.75681 & 133 & 141 \\
\hline 1 h after Epo & 10 & 138.8 & 1.988858 & 135 & 141 \\
\hline 1.5 h after Epo & 10 & 137.9 & 2.024846 & 135 & 141.5 \\
\hline 2 h after Epo & 10 & 137 & 3.496029 & 133 & 143 \\
\hline 1 h after L & 10 & 139.5 & 1.433721 & 138 & 142 \\
\hline 1.5 h after L & 10 & 137.85 & 2.147996 & 135 & 141.5 \\
\hline 2 h after L & 10 & 136.2 & 3.119829 & 131 & 141 \\
\hline
\end{tabular}

\section{Statistical Analysis}

Table 2 presents the (\%) hyponatremic influence of Epo regarding reoxygenation time. Also, Table 3 presents the (\%) hyponatremic influence of $\mathrm{U}-74389 \mathrm{G}$ regarding reperfusion time. Chi-square tests were applied using the ratios which produced the (\%) results per endpoint. The outcomes of chisquare tests are depicted at Table 4 . The statistical analysis was performed by Stata 6.0 software [Stata 6.0, StataCorp LP, Texas, USA].

\section{Results}

The successive application of chi-square tests revealed that U-74389G enhanced the hyponatremia by 1.695709 fold [1.323912-2.171914] more than Epo at $1 \mathrm{~h}$ ( $p$-value = $0.0000)$, by 0.8085706 -fold [0.7951-0.8222694] more than Epo at $1.5 \mathrm{~h}$ ( $p$-value $=0.0000$ ), by 3.008772 -fold [1.020358.866639] more than Epo at $2 \mathrm{~h}$ ( $\mathrm{p}$-value $=0.0455)$, more by 1.631842-fold [1.619651-1.644125] ( $p$-value $=0.0000)$ without drugs and by 2.74914-fold [2.74424-2.754048] more than Epo whether all variables have been considered ( $p$-value $=0.0000)$.

\section{Discussion}

The unique available study investigating the hyponatremic effect of U-74389G was the preliminary one [1]. Al-

Table 2: The (\%) hyponatremic influence of erythropoietin in connection with reperfusion time.

\begin{tabular}{|l|l|l|l|}
\hline Hyponatremia & \pm SD & Reperfusion time & p-value \\
\hline $0.72 \%$ & $\pm 2.07 \%$ & $1 \mathrm{~h}$ & 0.3054 \\
\hline $0.21 \%$ & $\pm 2.45 \%$ & $1.5 \mathrm{~h}$ & 0.7136 \\
\hline$-0.29 \%$ & $\pm 2.85 \%$ & $2 \mathrm{~h}$ & 0.7415 \\
\hline$-0.79 \%$ & $\pm 2.43 \%$ & Reperfusion & 0.1800 \\
\hline$-0.11 \%$ & $\pm 0.38 \%$ & Interaction & 0.7531 \\
\hline
\end{tabular}

Table 3: The (\%) hyponatremic influence of U-74389G in connection with reperfusion time.

\begin{tabular}{|l|l|l|l|}
\hline Hyponatremic & \pm SD & Reperfusion time & p-value \\
\hline $1.22 \%$ & $\pm 1.95 \%$ & $1 \mathrm{~h}$ & 0.0707 \\
\hline $0.17 \%$ & $\pm 2.74 \%$ & $1.5 \mathrm{~h}$ & 0.7753 \\
\hline$-0.87 \%$ & $\pm 3.31 \%$ & $2 \mathrm{~h}$ & 0.3995 \\
\hline$-1.29 \%$ & $\pm 1.97 \%$ & Reperfusion & 0.0176 \\
\hline$-0.32 \%$ & $\pm 0.36 \%$ & Interaction & 0.3693 \\
\hline
\end{tabular}

Table 4: The U-74389G/erythropoietin hyponatremic efficacies after chi-square tests application.

\begin{tabular}{|l|l|l|l|l|}
\hline Odds ratio & \multicolumn{2}{|l|}{ [95\% Conf. Interval] } & p-values & Endpoint \\
\hline 1.695709 & 1.323912 & 2.171914 & 0.0000 & $1 \mathrm{~h}$ \\
\hline 0.8085706 & 0.7951 & 0.8222694 & 0.0000 & $1.5 \mathrm{~h}$ \\
\hline 3.008772 & 1.02035 & 8.866639 & 0.0455 & $2 \mathrm{~h}$ \\
\hline 1.631842 & 1.619651 & 1.644125 & 0.0000 & Reperfusion \\
\hline 2.74914 & 2.74424 & 2.754048 & 0.0000 & Interaction \\
\hline
\end{tabular}


though the most famous activities of neuroprotection and membrane-stabilization properties, it accumulates in the cell membrane, protecting vascular endothelium from peroxidative damage but hardly penetrates the blood-brain barrier. It elicits a beneficial effect in ototoxicity and Duchenne muscular dystrophy. It increases ygt, superoxide dismutase (SOD) and glutathione (GSH) levels in oxygen-exposed cells. It treats septic states and acts as immunosuppressant in flap survival. It prevents the learning impairments, it delays the early synaptic transmission decay during hypoxia improving energetic state of neurons. It shows antiproliferative properties on brain cancer cells and is considered as a new promising anti inflammatory drug for the treatment of reperfusion syndrome in IR injuries. The same authors confirmed [2] the short-term hyponatremic effect of Epo preparations in non iron deficient individuals. Serum sodium levels at baseline are not available for many reasons: The first is that the baseline blood sampling of $2 \mathrm{cc}$ might be ominous for the small rats so as the experiment jeopardize not to proceed. The second is that we should alike to receive baseline histologic samples, as the experiment ended up to internal genitalia study, something which was impossible. The third is that such baseline values would be deprieved by the "IR event" which we wished to investigate whether and how much would be reverted by the drugs. These are the reasons we used as baseline values those of placebo groups.

Liu L, et al. [3] identified Epo as the predominant treatment component; with sodium selenite serving as an adjuvant, and combination treatment was markedly more effective, compared with either treating drug alone. The optimal ratio of treatment was 10: 1 (10 IU EPO: $1 \mu$ g sodium selenite). Together, the results suggested that co-administration of EPO and sodium selenite effectively ameliorates IRI-induced renal injury by reducing oxidative stress and activating the PI3K/NO signaling pathway in renal IRI. Jeong JH, et al. [4] confirmed the neuroprotective effects of recombinant human erythropoietin (rhEPO)-loaded poly (lactic-co-glycolic acid) (PLGA) nanoparticles stabilized by sodium cholate (rhEPO-Ch-NP) and compared their effects with those of rhEPO. Notably, it was demonstrated that rhEPO-Ch-NPs were safer at any concentration investigated and rescued more neuronal cells in an in vitro model of cerebral ischemia. Deliyanti D, et al. [5] found that a low-salt (LS) diet reduced erythropoietin, mineralocorticoid receptor, angiotensin type 1 receptor and renin mRNA levels in retina, whereas, as expected, plasma levels of aldosterone and renin were increased. In cultured Müller cells, high salt increased epithelial sodium channel alpha $(E N a C \alpha)$, which was prevented by mineralocorticoid receptor and angiotensin type 1 receptor blockade in pregnant Sprague Dawley rats. Kang J, et al. [6] significantly up-regulated the expression of protein and mRNA levels of HIF-1 $\alpha$, VEGF and EPO in the $\beta$-sodium aescinate $(S A)$ group $(P<0.05)$ after return of spontaneous circulation in rat cerebral cortex. Bimpis A, et al. [7] revealed an injury specific type of behavior after U-74389G administration that could be considered as neuroprotective since $\mathrm{Na}(+), \mathrm{K}(+)$ - and $\mathrm{Mg}(2+)$-ATPase inhibition might in this case diminish the local ATP consumption in a spontaneous intracerebral hemorrhage (ICH) porcine model. Vignes JR, et al. [8] found that lazaroid compounds and certainly U-74500A decreased neuronal death to 37$23.5 \%$, U-74389G to $37-32 \%$, and U-83836E to $42-33 \%$ compared with exposed primary cultures of cortical neurons in rats exposed to $0.5 \mathrm{mM}$ sodium cyanide for $6 \mathrm{~h}$. Washo-Stultz $\mathrm{D}$, et al. [9] assumed that damage to mitochondria is an upstream event in sodium deoxycholate ( $\mathrm{NaDOC}$ )-induced apoptosis and that a pro-oxidant state of the cell favors survival. Lazaroid pre-treatment caused a marked decrease in $\mathrm{NaDOC}$-induced activation of the anti-apoptotic transcription factor, NF-KB, which may provide the basis for the sensitization to apoptosis caused by these antioxidants. Horáková L, et al. [10] found that $U 74389 \mathrm{G}$ decreased the preventive effect concerning lipid peroxidation by (160 IC50 in $\mu \mathrm{mol} / \mathrm{l}$ ) in brain homogenate. Stanimirovic DB, et al. [11] implicated both the inhibition of $\mathrm{Na}, \mathrm{K}$-ATPase and membrane lipid peroxidation in the presence of the steroid antioxidants U-74500A and $\mathrm{U}-74389 \mathrm{G}(5-20 \mu \mathrm{M})$ in rat cerebromicrovascular endothelial cells.

According to above, Table 4 shows that U-74389G has 2.74914-fold [2.74424-2.754048] more hyponatremic effect than Epo ( $p$-value $=0.0000)$ whether all variables have been considered ( $p$-value $=0.0000) ;$ a trend accentuated along time, in Epo non-deficient rats.

\section{Conclusion}

The anti-oxidant agent U-74389G was proved having 2.74914-fold [2.74424-2.754048] more hyponatremic effect than Epo whether all variables have been considered ( $p$-value $=0.0000) ;$ a trend accentuated along the short term time frame of the experiment in rats. A biochemical investigation remains about how U-74389G mediates in these actions. The mechanism of hyponatremia is impossible by the current knowledge. It seems that sodium somewhere is consumed; however, further assumptions about the hyponatremic trend is high risky. This is the reason that this study is statistical and not molecular, nevertheless, the authors finally recommend for a further molecular elucidation of hyponatremia.

\section{Acknowledgement}

Acknowledged in preliminary studies.

\section{Ethical Approval}

"All applicable international, national, and/or institutional guidelines for the care and use of animals were followed".

\section{References}

1. Tsompos C, Panoulis C, Toutouzas K, et al. (2014) The effect of the antioxidant drug "U-74389G" on sodium levels during ischemia reperfusion injury in rats. Crit Care \& Shock 2: 31-36.

2. Tsompos C, Panoulis C, Toutouzas K, et al. (2015) The effect of erythropoietin on sodium during ischemia reperfusion injury in rats. J Clin Anal Med 6: 569-572.

3. Liu L, Liu C, Hou L, et al. (2015) Protection against ischemia/ reperfusion-induced renal injury by co-treatment with erythropoietin and sodium selenite. Mol Med Rep 12: 79337940.

4. Jeong JH, Kang SH, Kim JH, et al. (2014) Protective effects of poly (lactic-co-glycolic acid) nanoparticles loaded with erythropoietin 
stabilized by sodium cholate against glutamate-induced neurotoxicity. J Nanosci Nanotechnol 14: 8365-8371.

5. Deliyanti D, Armani R, Casely D, et al. (2014) Retinal vasculopathy is reduced by dietary salt restriction: Involvement of $\mathrm{Glia}, \mathrm{ENaC} \alpha$, and the renin-angiotensin-aldosterone system. Arterioscler Thromb Vasc Biol 34: 2033-2041.

6. Kang J, Gong P, Ren YB, et al. (2013) Effect of $\beta$-sodium aescinate on hypoxia-inducible factor- $1 \alpha$ expression in rat brain cortex after cardiopulmonary resuscitation. World J Emerg Med 4: 6368.

7. Bimpis A, Papalois A, Tsakiris S, et al. (2013) Modulation of crucial adenosinetriphosphatase activities due to U-74389G administration in a porcine model of intracerebral hemorrhage. Metab Brain Dis 28: 439-446.
8. Vignes JR, Hugon J (2006) In vitro efficacy of three lazaroids in a model of acute chemical neuronal hypoxia. Neurosci Lett 407: 171-175.

9. Washo-Stultz D, Crowley-Weber CL, Dvorakova K, et al. (2002) Role of mitochondrial complexes I and II, reactive oxygen species and arachidonic acid metabolism in deoxycholate-induced apoptosis. Cancer Lett 177: 129-144.

10. Horáková L, Ondrejicková O, Bachratá K, et al. (2000) Preventive effect of several antioxidants after oxidative stress on rat brain homogenates. Gen Physiol Biophys 19: 195-205.

11. Stanimirovic DB, Wong J, Ball R, et al. (1995) Free radical-induced endothelial membrane dysfunction at the site of blood-brain barrier: Relationship between lipid peroxidation, Na,K-ATPase activity, and 51Cr release. Neurochem Res 20: 1417-1427. 